\title{
Paresia de VI nervo congênita
}

\section{Congenital $V \mathcal{I}$ nerve palsy}

$\begin{array}{ccc}\text { Marcelo } & \text { Francisco Gaal } & \text { Vadas }^{1} \\ \text { Ronaldo } & \text { Boaventura } & \text { Barcellos }^{1}\end{array}$

${ }^{1}$ Pós-Graduando Doutorado do Depto de Oftalmologia do Hospital das Clínicas da Faculdade de Medicina da Universidade de São Paulo. Médico do Setor de Estrabismo do Departamento de Oftalmologia da Santa Casa de São Paulo.

Endereço para correspondência: Calçada das Draçenas, 10 - Alphaville - Barueri (SP) CEP 06453-000. E-mail:mvadas@aol.com

\begin{tabular}{|l|}
\hline R E S U м о \\
\hline Os autores relatam caso de uma criança de 2 anos com hipótese diagnóstica \\
de paresia de VI nervo esquerdo, congênita. O paciente foi submetido a \\
cirurgia no olho esquerdo, com amplo retrocesso do reto medial e grande \\
ressecção do reto lateral, sendo que as características peroperatórias e \\
resultado cirúrgico reforçam a hipótese diagnóstica inicial.
\end{tabular}

Descritores: Paralisia/congênito; Doenças dos nervos cranianos; Diagnóstico diferencial; Relato de caso

\section{INTRODUÇÃO}

A paresia de VI nervo é afecção bastante freqüente dentre as paresias oculomotoras, entretanto, as congênitas são pouco freqüentes ${ }^{(1-3)}$. Devese atentar para o diagnóstico diferencial com a síndrome de Duane tipo I que apresenta somente alterações discretas da rima palpebral, com a síndrome de Ciancia muito assimétrica e com a síndrome de Moebius.

A paresia pode ser congênita, devido a alteração do desenvolvimento do núcleo ou de fibras motoras do nervo, ou adquirida. O longo trajeto do nervo, que surge do assoalho do IV ventrículo na ponte, contorna o colículo facial, emerge do sulco bulbo-pontino, penetra o seio cavernoso e entra na órbita através da fissura orbital superior, torna-o susceptível a variados insultos, quer sejam traumáticos, inflamatórios, vasculares ou tumorais. Em adultos, deve-se considerar em primeiro lugar causa microvascular, já em crianças, neoplasia (sobretudo glioma de tronco), trauma e causas inflamatórias ${ }^{(4)}$. Dentre as causas inflamatórias, destaca-se a síndrome de Gradenigo: paresia ou paralisia do VI nervo e nevralgia do trigêmio, decorrentes da propagação da inflamação causada por otite ou mastoidite.

\section{RELATO DE CASO}

PHJM, 2 a 3m, sexo masculino, procedente da Bahia, com história de desvio do olho esquerdo para dentro desde o nascimento.

Antecedentes Pessoais: Criança eutrófica, criptorquidia esquerda.

Antecedentes Familiares e Oculares: ndn.

O exame oftalmológico revelou baixa hipermetropia na refração estática (+1,50 DE em ambos os olhos), oftalmoscopia e biomicroscopia normais em ambos os olhos.

Quanto às condições motoras, ao Hirschberg, fixando OD (Figura 1):
OT

(dextroversão)
ET 40 DP

(PPO)
$\mathrm{ET}>80 \mathrm{DP}$

(levoversão) 

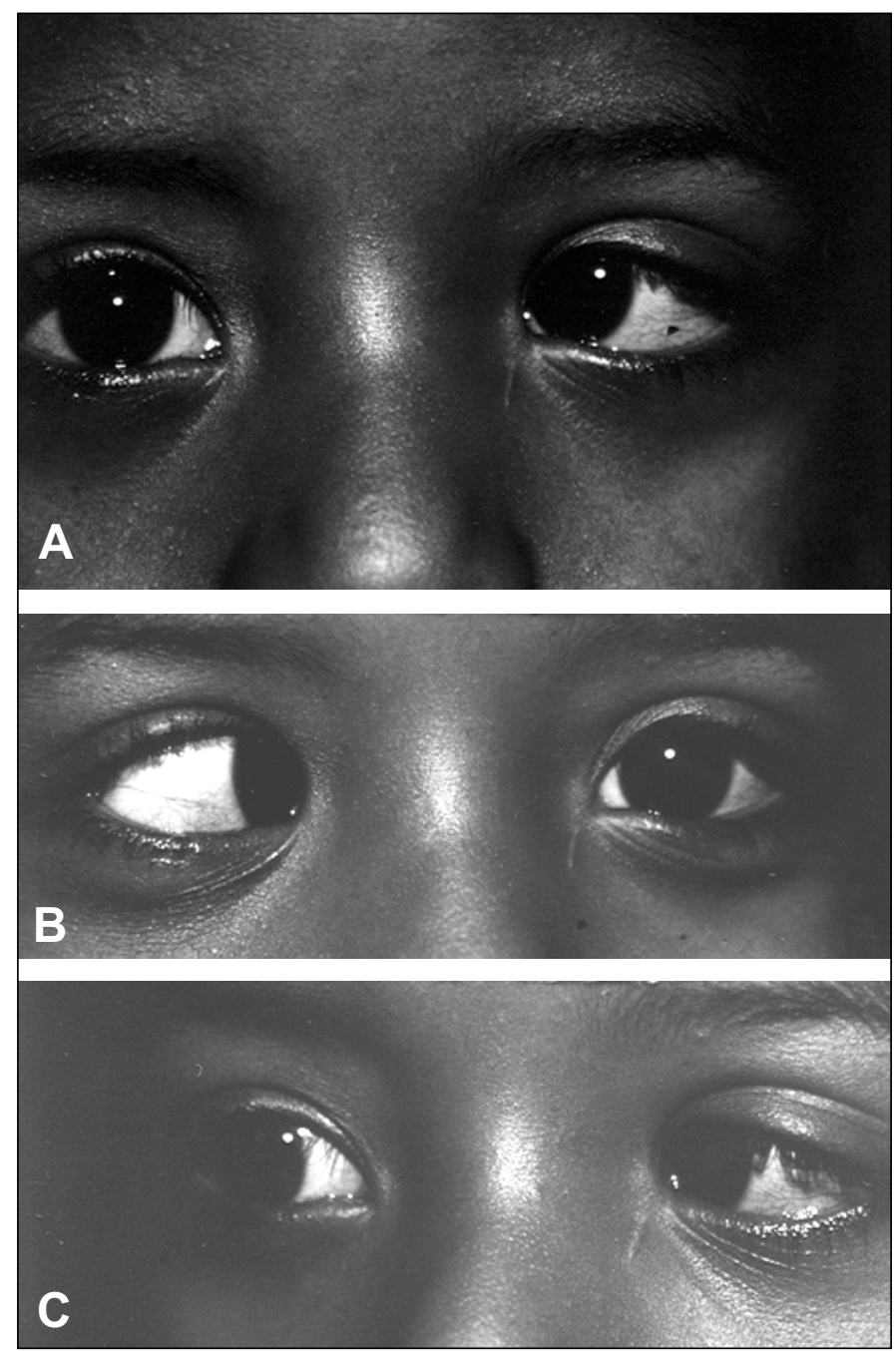

Figura 1 - Pré-operatório: A) posição primária do olhar; B) levoversão; C) dextroversão

Fixação binocular: Prefere OD. Quando fixa com olho esquerdo (OE) desvio aumenta (ET 60 DP). Às versões e ducções, marcada limitação de levoversão e abdução do olho esquerdo (-4). Rima palbebral normal em todos os movimentos do olhar.

Iniciado tratamento oclusivo, alternado, por aproximadamente 1 ano, quando então submeteu-se a cirurgia para exploração peroperatória do OE e correção do estrabismo. Utilizou-se relaxante muscular não despolarizante (Atracurium $0,3 \mathrm{mg} / \mathrm{kg}$ ) na anestesia geral. O teste de ducção passiva mostrou-se discretamente positivo. O músculo reto medial estava com aspecto normal, porém no teste de Queré, quando desinserido, atingiu somente a meia distância entre o limbo e o centro da córnea. Notou-se também aspecto normal da esclera subjacente. Foi então retrocedido $8 \mathrm{~mm}$. O reto lateral, por sua vez, mostrou-se fino e alongado sendo então ressecado $10 \mathrm{~mm}$.

Uma semana após a cirurgia, paciente próximo da ortotropia em PPO (ET 5DP) e com a abdução francamente mais livre (-2), estando a adução pouco comprometida (-1), como podemos evidenciar na figura 2 .
XT 10 DP
ET 5 DP
(PP0)
ET 20 DP
(dextroversão)
(levoversão)

Esta disposição mostrou-se estável ao longo do tempo no PO 6 meses (Figura 3).

$\begin{array}{ccc}\text { XT 10 DP } & \text { ET 5 DP } & \text { ET 20 DP } \\ \text { (dextroversão) } & \text { (PP0) } & \text { (levoversão) }\end{array}$

DISCUSSÃO

$\mathrm{O}$ caso descrito nos pareceu estar enquadrado dentro das paresias congênitas do VI nervo, apesar da raridade desta doença ${ }^{(5)}$. Não apresenta alterações da rima palpebral ou cocontração em adução o que tende a afastar a possibilidade de Síndrome de Duane. O achado peroperatório de discreta contratura do reto medial sem afinamento escleral subjacente e ainda, o fato de termos ressecado amplamente o reto lateral e retrocedido amplamente o reto medial - proposições equivocadas na Síndrome de Duane por aumentar a cocontração e poder causar divergência sinérgica ${ }^{(6)}$ - corroboram esta idéia. O grande desvio em PPO também não é freqüente na Síndrome de Duane. A ausência de torcicolo, nistagmo latente ou DVD aliado também aos achados peroperatórios (músculo reto medial contraturado) e pós-operatórios (bom resultado com retrocesso/ressecção monocular) tendem também a afastar a possibilidade de Síndrome de Ciancia assimétrica.
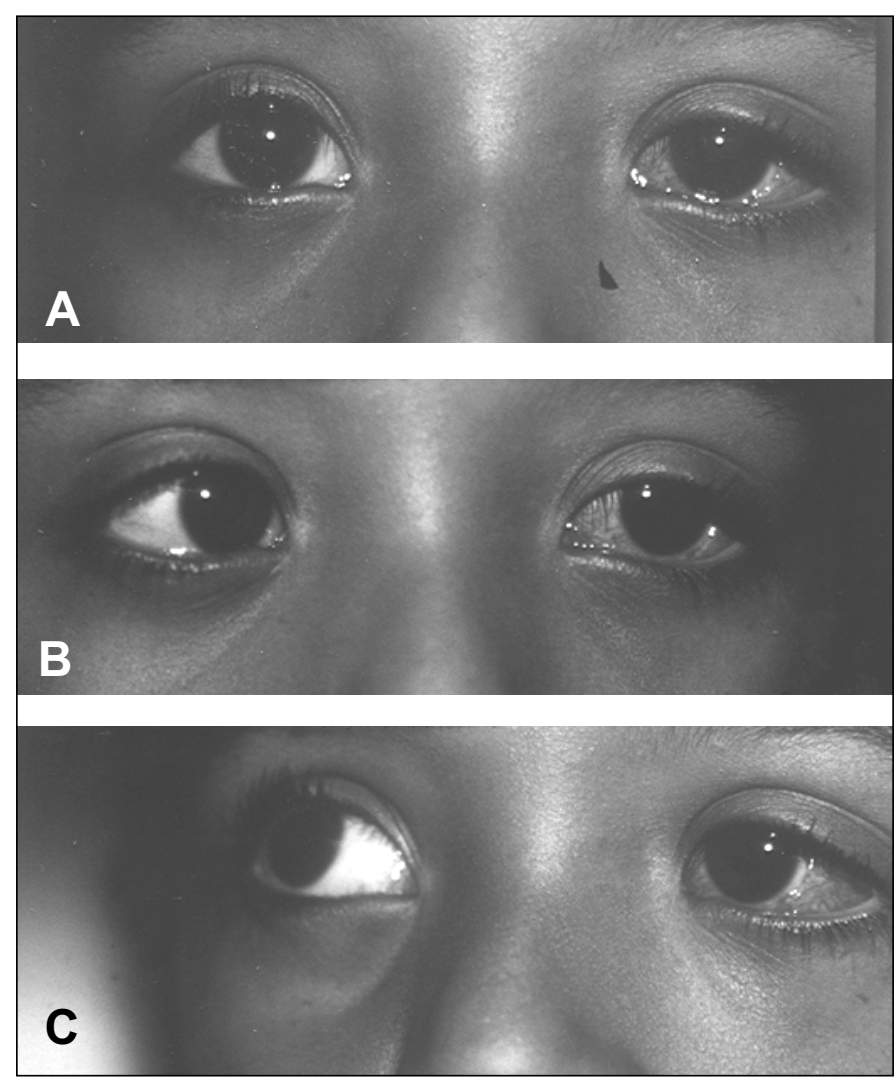

Figura 2 - PO 1 semana: A) posição primária do olhar; B) levoversão; C) dextroversão 

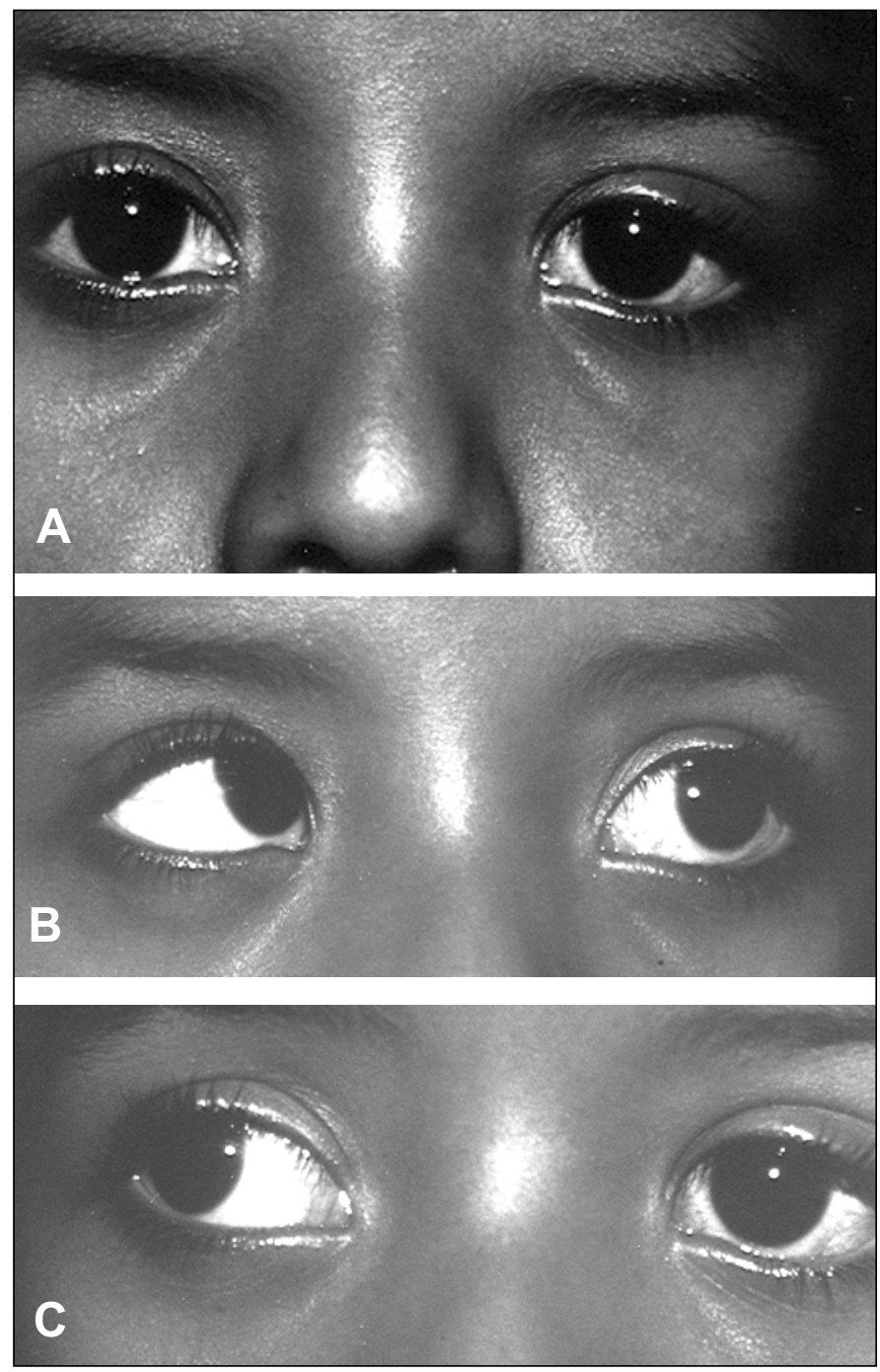

Figura 3 - PO 6 meses: A) posição primária do olhar; B) levoversão; C) dextroversão

Surpreendeu-nos a discreta limitação de adução obtida apesar da grande magnitude do retrocesso/ressecção e da evidente melhora da abdução, supostamente pela liberação da contenção que o reto medial representava. A incomitância do quadro, apesar do tempo transcorrido, já foi observado como sendo comum nas paresias de VI congênitas ${ }^{(7)}$.
A idade do paciente não nos permitiu realizar de forma conclusiva manobras pré-operatórias, como o teste de velocidade sacádica de abdução. $\mathrm{O}$ teste de forças geradas e outras manobras pré-operatórias com pinça também não foram realizadas. Entretanto, o bom resultado cirúrgico obtido através de amplo retrocesso/ressecção sugere tratar-se de paresia e não paralisia ${ }^{(8)}$.

Baseado no risco relativamente alto de neoplasia ${ }^{(1,9)}$, devese rastrear por neuroimagem a população infantil acometida de paresia de VI nervo, mesmo sendo isolada e sobretudo nos casos em que o início da doença não está claro.

\section{ABS T RAC T}

The authors report a case of a 2-year-old child with a probable diagnosis of congenital left VI nerve palsy. Surgical correction was performed OS: large medial rectus recession and large lateral rectus resection were carried out. Findings during surgery and final outcome support the diagnosis of congenital VI nerve palsy.

Keywords: Paralysis/congenital; Cranial nerve diseases; Differential diagnosis; Case report

\section{REFERENCIAS}

1. Holmes JM, Mutyala S, Maus TL, Grill R, Hodge DO, Gray DT. Pediatric third, fourth, and sixth nerve palsies: a population-based study. Am J Ophthalmol 1999;127:388-92.

2. Ernest JT, Costenbader FD. Lateral rectus muscle palsy. Am J Ophthalmol 1968;65:721-6.

3. Afifi AK, Bell WE, Menezes AH. Etiology of lateral rectus palsy in infancy and childhood. J Child Neurol 1992;7:295-9.

4. Lee MS, Galetta SL, Volpe NJ, Liu GT. Sixth nerve palsies in children. Pediatr Neurol 1999;20:49-52.

5. Prieto-Diaz J, Souza-Dias C. Las paresias y paralisis oculomotoras. In: PrietoDiaz J, Souza-Dias C. Estrabismo. La Plata: Editora J. Poch; 1996. p. 405-14.

6. Collins CC, Jampolsky A, Howe PS. The unique medial rectus characteristics in Duane's Syndrome. In: XII Congresso do Conselho Latino-Americano de Estrabismo; 1996 Maio 9-11, Buenos Aires, Argentina; 1996. p. 569-76.

7. Parks MM, Mitchell PR. Cranial nerve palsies. In: Tasman W, Jaeger EA. Duane's clinical ophthalmology [monografia em CD-ROM]. Philadelphia, J. B. Lippincott; 1999.

8. Rosenbaum AL. Management of acute and chronic VI nerve palsy. In: XII Congresso do Conselho Latino-Americano de Estrabismo; 1996 Maio 9-11, Buenos Aires, Argentina; 1996. p. 373-6.

9. Aroichane M, Repka MX. Outcome of sixth nerve palsy or paresis in young children. J Pediatr Ophthalmol Strabismus 1995;32:152-6.

\section{Novidades}

\section{Internet!!!}

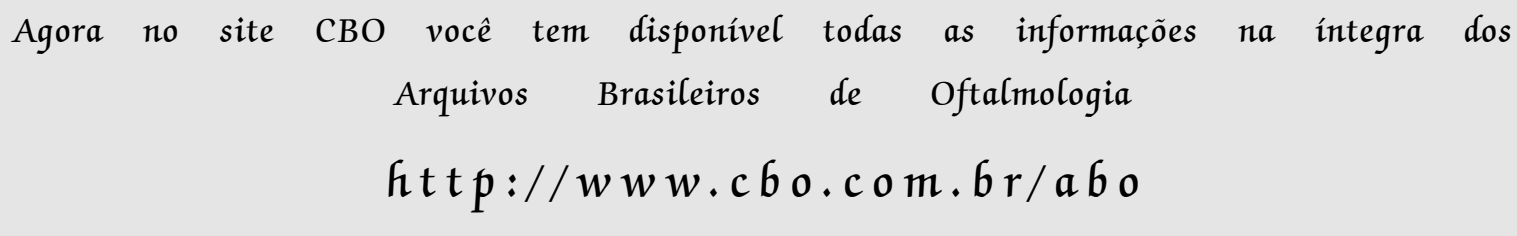

